\title{
Customer Churn Prediction using Predictive Analytics in Telecommunication Market: A Review
}

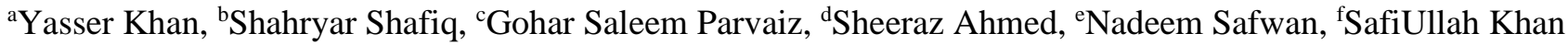 \\ a,b,d,e Iqra National University, Peshawar Pakistan \\ 'Institute of Management Sciences Peshawar \\ fPakistan Telecommunication Company Limited \\ Corresponding Author: yss_kh@yahoo.com
}

\begin{abstract}
In the face of extreme competitive telecommunication market, the cost of acquiring new customer is much more expensive than to retain the existing customer. Therefore, it has become imperative to pay much attention towards retaining the existing customers in order to get stabilize in market comprised of vibrant service providers. In current market, a number of prevailing statistical techniques for customer churn management are replaced by more machine learning and predictive analysis techniques. This article reviews the customer churn prediction problem, factors escalating the phenomena, prediction through predictive analytics, steps for processing of predictive analytics and evaluation of performance metrics for various churn prediction models are surveyed. Moreover, the CRM data from Pakistan Telecommunication Company limited as case study to discuss the process of data mining and predictive analytics for customer churn prediction.
\end{abstract}

Keywords- Predictive analytics, Telecom customer churn and retention, customer relationship management

Date Received 20-09-2019

Date Accepted 25-12-2019

Date Published 31-12-2019

\section{INTRODUCTION}

$\mathrm{T}$ HE public services has improved significantly on the emergence of mobile phone technology and delivered benefits to common citizen in term of connectivity, access to markets and social integration. This trends in this latest technology, however, has brought a score of demands and complaints regarding service as well [1].

In today competitive world of telecommunication technology, the surviving of operators is quite difficult due to frequent movement of customers who are discarding a particular service on basis of dissatisfaction or acquiring of offering from another service provider. Technically, this phenomena is termed as churn that has help customer obtained better service on one hand but lead significant revenue or profit loss to that network providers invested in one of expensive market. The re-acquisition of this defected customer and many more is big challenge for the company to strengthen its position in market. Therefore, companies are applying all possible efforts by applying cutting edge technology and computer application in advance to highlight those customers likely to switch over to their competitors and prevent themselves from significant loss in term of potential revenue/profit loss. So, the entire process of identification of dissatisfied customer is called churn prediction. Therefore, computerized search is necessary to highlight those customers in advance intending to end the subscription to an operator and initiate retention program [2]. By customer churn prediction, the company is assigning probability to customers on the basis of historical record and future churning behavior before it actually exhibit high propensity to switch over to competitor. Some company are also ranking their subscribers into category from high to low likely churn customers and marketing team is involved to start retention campaign to high risk customer. The behavior of potential churn customers can be modeled as most susceptible to marketing campaign and those who will churn anyway, whether they are subject to extensive marketing actions are termed as persuadable and non-persuadable customers respectively and the phenomena is known as net effect or uplift modeling [3]. The churn can be reduced by offering all possible features possible in a particular service or product and giving customer centric approach by revising all traditional strategies to face the fierce competition in tough market operating under dynamic condition with competitive offerings [4].

The identification of potential churn customers by application of predictive analytics can add substantial amount of profits compare to random selections of these customers so, wastage of resources are controlled and targeted retention 
campaign are taken place [5]. This is imperative to highlight that profit varies according to technique used for predictive analysis which is clearly evident from one of study conducted on $10 \%$ customers of company out of total 5 million customer are passed through retention campaign which profit has climbed to hundred thousand dollars by simply changing the prediction technique [6].

\section{LITERATURE REVIEW}

The behavior of video game players is predicted and modeled through data acquired in the form of huge data set by games developers. The video gaming has revolutionize the industry but churn of player is posing a big threat that is badly impacting the monetization of games. The modeling method is capable for prediction of churn from beginner level of player to maturity and ultimately leave the gaming industry. This method is applicable to score of variables besides gaming industry and help in online forecasting for parallelization of algorithms [7]. In contrast to conventional method for churn prediction methods normally used for telephone service provider in saturate telecom market, the model is devised and focused on profit maximization by applying new technique termed as expected maximum profit for customer churn. The technique is strengthen through application of profLogit classifiers that is similar to logistic model and genetic algorithm. The model has attained excellent position in profit based precision for nine real life data sets [8].The customer retention has significantly improved both strategically and analytically despite fierce competition that has allowed to emerge new system CRM (Customer Relationship Management). The big advantage currently provided by CRM is classification and prediction of potential customers about to leave the company from big data set. Despite the fact that several models were developed for customer retention but all have some serious limitation and remain unable to determine the accurate and precise prediction. The main reason behind the false result in previous model is due to application of statistical technique (although successful in diverse domain), validation through benchmarking of data sets having noisy and missing values in data, true positive(TP) is totally ignored which classified between churners and nonchurners, non-application of fuzzy logics [9].

By applying relational learner and employing social network analytics to determine the customer churn prediction from nine data sets obtained has significantly improved the performance of predictive modeling. The statistical evaluation of relational learners and model accuracy in combination with traditional methods, network construction and application of weights and edges in network for optimal prediction of churn in saturated telecom industry [10].The impact service quality, price and convenience on of customer satisfaction in one study by taking the response from on questionnaire drawn on five point likert scale. The customer satisfaction was modeled with different customer segments to determine the customer behavior. The conclusion found was that customer retention mainly depend on socio-demographic characteristic and customer satisfaction. Also, declared the customer behavior as immature until the industry has not finished evolution stage [11].The relationship of subscriber churn and customer loyalty is determined in another study in Korean telecommunication market by applying binomial logic model. The variables used in study are call quality, brand image, income level, call quality and the period the customer remain in subscript with a company to seek the switching behavior of customers [12].In another study the author has collected the responses from over 480 customers of telecom industry in Taiwan where the relationship of CRM (customer relationship management) and customer loyalty is established with controlling variable of brand image. Also, demographic information is also collected. The research is concluded on strong positive correlation between customer relationship, brand image and loyalty [13].

Similarly, in pursuit of factors affecting customer churn in telecom sector, the relationship of customer service and customer retention is formed with mediating effect of customer satisfaction. The data from Nigerian mobile service provider was collected and analyzed to predict the perceived value, customer service and satisfaction in order to predict the customer behavior. The result clearly reveal that if customer are provided with quality of service, they will continue their relationship with existing network and would return if they get defected by chance [14]. By taking other variables like price, facility, communication, customer service and customer satisfaction to find the customer perception to choose which mobile service is likely to fulfill the need of the customer was research conducted in India. The model was constructed having three major variable customer care (depend upon complaint management system), price (depend upon promotional offers, call rate and product range), service provision (depend upon call connectivity, call quality and drop rat in a specific geographical area). The author concluded the research by declaring service provision and then price are main factors greatly impact customer decision and lastly the customer care [15].The switching behavior to competitor was analyzed in another study conducted at Chennai, India that influence customer decision. Author has made good attempt in his article to prove that churning decision from a particular service provider were not influence by advertisement campaign rather family decision. The amount of family decision to switch over is extracted as $41 \%$ as compared to only $2 \%$ by advertisement. Although others factors influence over all process are service problem, customer service, service usage and cost. He also declared call rate as important decision making variable after network coverage [16]. The time for telemarketing is decisive factors for customer retention especially when the contract between service provider and customer is near to expire. The author, however, declared varies from one group to another group during his studies. The samples were taken in different groups depend on monthly billing charges, hence almost 400 customers were randomly selected and research is concluded [17]. The switching behavior was further studied taking service quality, customer demographics, customer satisfaction and relational investment as independent variables impacting 
customer churn. The behavior was also researched with mobile network portability (MNP) which is positively affecting the churning behavior [18].

In another research analysis, almost 1000 prepaid subscribers underwent the studies from Andhra Pradesh, India, used demographic profiles, monthly billing, monthly usage, handset used and customer satisfaction. The statistical analysis conducted by author are independent sample T-Test and Discriminant analysis reveal the direct impact of call tariffs, customer care, network coverage on churning behavior of customer [19]. In order to determine the churning impact, the sixteen years historical data obtained from Newspaper Company was considered for predicting the churn. The relationship of socio demographic, frequency, fee and duration related parameters were established with churn by using classification and logistic regression [20].

\section{PREDICTIVE ANALYTICS OVERVIEW}

This technique is basically used to extracts hidden data patterns from big data bases so briefly know as knowledge discovery in data base. DM is primarily comprised of knowledge system derived from data base systems, machine learning, statistics and artificial intelligence and can explained as identification of interesting patterns and transformation into understandable manner in large data base.

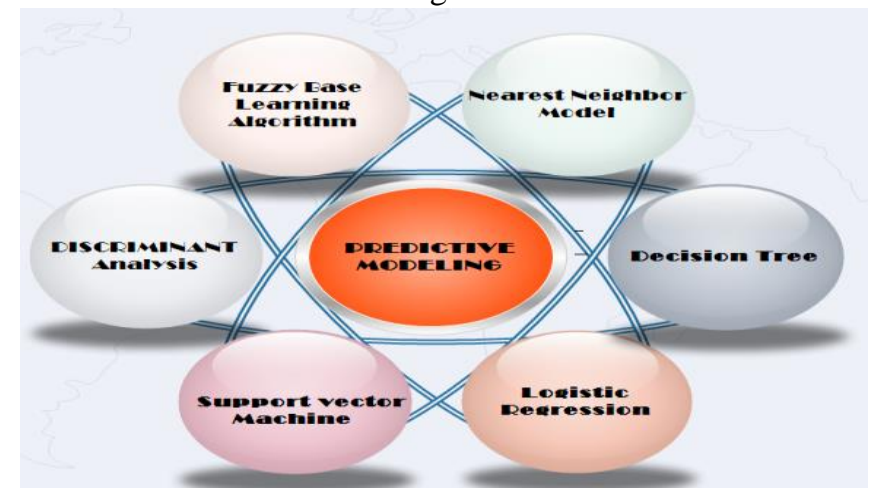

Fig. 1. The Predictive Modeling Techniques

Firstly, the data base system are varied according to function, they performing in data mining approach like spatial, multimedia, legacy, web, relational and transaction data base. Secondly, categorization of data mining is according to techniques used for data mining like data drive, autonomous knowledge, interactive, generalized, mathematical-based, pattern based and underlying data mining approach. Thirdly DM can be categorized on the basis of knowledge. Below are most famous technique used are Association, Clustering, prediction and sequential patterns. In association, each data item is classified into predefined sets of groups, clustering makes classification according similar characteristics items, prediction is used to established connection between dependent

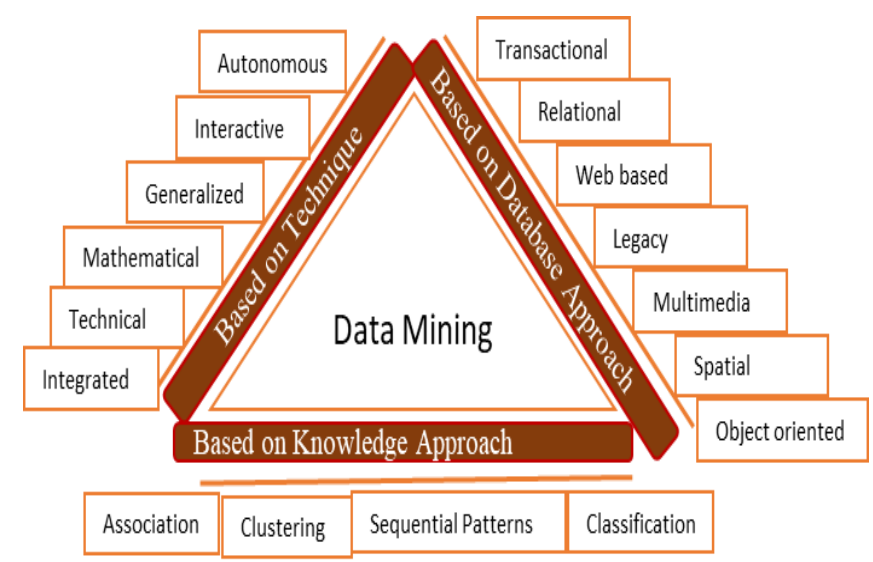

and independent variables while sequential patters seeks to highlight same pattern data or transaction data over specific period of time.

Fig. 2. Different Approaches used for Data Mining

TABLE 1: METHODS OF DATA MINING

\begin{tabular}{|c|c|c|}
\hline Function & Methods & Segmentation \\
\hline \multirow{4}{*}{ Classification } & Decision Tree & \\
\hline & Fuzzy Logic & Credit Scoring \\
\hline & Neural Network & Market Segmentation \\
\hline & Genetic Algorithm & \\
\hline \multirow{3}{*}{ Estimation } & Statistics & \\
\hline & Time Series & $\begin{array}{l}\text { Exchange rate } \\
\text { estimation }\end{array}$ \\
\hline & Neural Network & \\
\hline \multirow{4}{*}{ Segmentation } & Decision Tree & \\
\hline & Statistics & Market Segmentation \\
\hline & Neural Network & \\
\hline & Genetic Algorithm & \\
\hline \multirow{3}{*}{ Association } & Set Theory & \\
\hline & Statistics & Cross Selling \\
\hline & $\begin{array}{l}\text { Bayesian } \\
\text { Classification }\end{array}$ & \\
\hline \multirow{3}{*}{ Prediction } & Decision Tree & \\
\hline & Neural Network & Fraudster Prediction \\
\hline & Regression & Churn Prediction \\
\hline
\end{tabular}




\section{PREDICTIVE ANALYTICS TECHNIQUES}

\section{A. Decision Tree}

For classification of customer churn problem, this method is most commonly used. The customer data set is classified by continuously dividing the tree until reach at leaf node. Hence the rule of divide and conquer is used in the development of decision tree is used so by evaluation the record of customers obtained from a specific telecom operator when reach to leaf node would complete the process of decision tree. The technique although has some limitation in addressing complex and non-linear connection in attributes. In order to enhance the performance of the algorithm, the method is frequently used in combination with other techniques like with neural network. . The minor causes for customer churn is to be ignored while the area of subscribers is the main feature for classification hence application of decision tree for classification is used to determine the customer churn analysis in telecommunication industry [21].

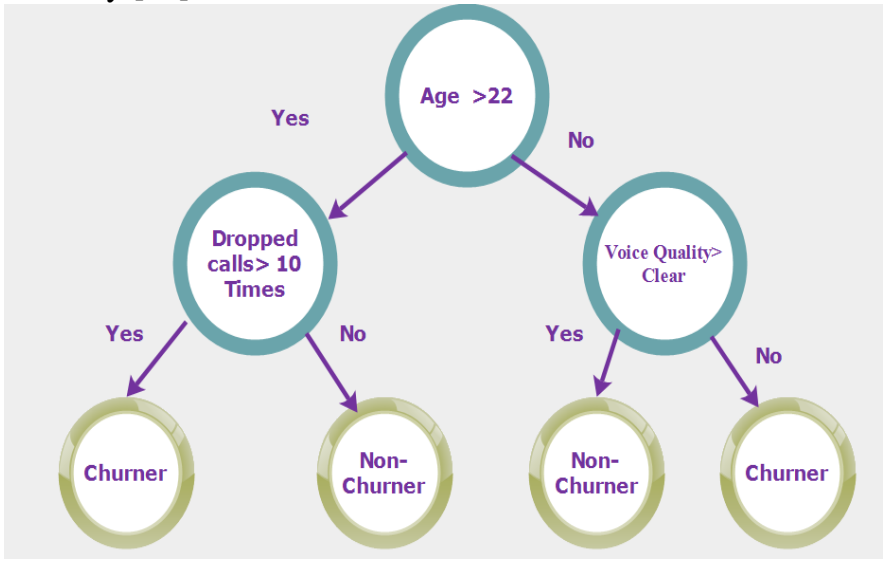

Fig. 3. Decision Tree for Classification

\section{B. Logistic Regression}

The conditional or posterior probability of discrete value is computed for generation of continuous or discrete variables. The standard error rate is calculated for each variable in this modeling. On the basis of significance to linear regression for churn prediction LG model is designed and constructed. The main aim behind logistic regression is identify either the customer is going to be churner or non-churner

Logistic regression

$$
\begin{aligned}
& =\log \left[\frac{\pi i}{(1-\pi i)}\right] \\
& =\alpha 0+\alpha 1 X i 1+\alpha 2 X i 2+\cdots \ldots .+\alpha k X i k
\end{aligned}
$$

Where $\pi_{\mathrm{i}}$ is the outcome probability,

$\alpha_{0}, \alpha_{1}, \alpha_{2} \ldots \alpha_{k} \quad$ are the co-efficient

(Transformed into odd rations with importance degree)
And $\mathrm{X} 1, \mathrm{X} 2 \ldots ., \mathrm{Xik}$ are the predictive variables

\section{Support Vector Machine}

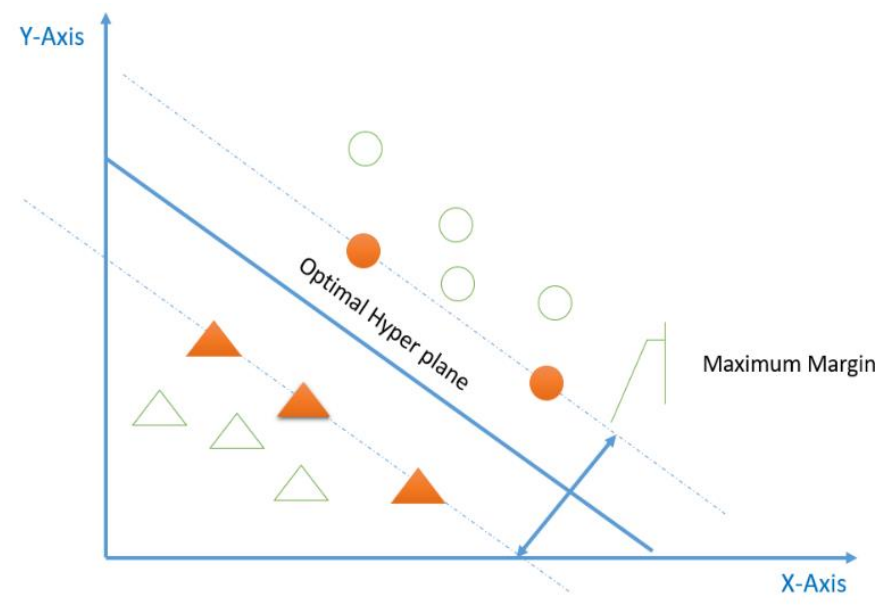

Fig. 4. The Support Vector Machine

This technique is design mainly to reduce structural risk minimization, are developed by Boser, Guyon and Vapnik . This is supervised learning mechanism which recognized and analyzed the data set patterns for classification and regression problems.

The performance of the SVM is improved through introduction of Kernel function and a lot of emphasis is laid to develop best kernels or combination of kernels. For best data transformation, the SVM sometime surpass both neural network and Decision Tree in performance.

\section{Nearest Neighbor Algorithm}

The technique mostly used in data mining algorithm for predictive analytics in classification problems, estimation and prediction. In K-Nearest Neighbor, J determine the number of variables used in neighborhood. The two famous techniques used are distance function and cardinality.

$$
\text { Distance function } \mathbf{y}_{\mathbf{i}}=\frac{1 \sum_{\mathrm{xjeN}(\mathrm{xi}) \mathrm{yj}}}{\mathrm{k}}
$$

The neighborhood of $\mathrm{x}$ corresponds to $\mathrm{xi}, \mathrm{N}(\mathrm{xi})$ and $\mathrm{k}$ is the constant of proportionality

In Cardinality " $\mathrm{k}$ " determine the complexity and importance of nearest neighbor function, whenever the $\mathrm{k}$ is higher in number the model may be considered as less adaptive and another circumstances k- value is used for goodness of fit test.

\section{E. Discriminant Analysis}

The technique most commonly used for linear modelling in patter recognition and machine learning technique for convergence and divergence of objects categories. Discriminant analysis is also frequency used to find the variables that separate two or more than two variables. The DA (Discriminate 
analysis) function used to separate the variables into two groups are as below:

$$
p \frac{1}{x}=\frac{1}{1+\left(e^{\alpha+\beta x}\right)^{-1}}
$$

Where

$$
\beta=\sum(\mu 1-\mu 0)
$$

$$
\alpha=-\log \frac{\eta 1}{\eta 0}+\frac{1}{2}(\mu 1+\mu 0) \sum(\mu 1-\mu 0)
$$

Where $\pi 0$ and $\pi 1$ are called prior probabilities, $\mu 0$ and $\mu 1$ are the distribution mean

\section{F. Fuzzy Base Learning Algorithm}

This is the most flexible, assist in modeling of non-linear function and tolerant to data which may be imprecise. This can help in implementation of conventional control techniques.

\section{G. Neural Network}

For the resolution of complex and non-linear problem, this technique has gained very much popularity. This can be both represented in hardware- based such as neuron are represented as physical components or as computer model in software form. The technique due to extensive structure has been using variety of learning algorithm and topologies. In supervised learning, when more than one hidden layers are used then model is defined as multilayer perceptron can train a scores of back propagation algorithm (BPN). Although complex shape but artificial neural network outperformed both logistic regression and C5.0 for customer churn prediction.

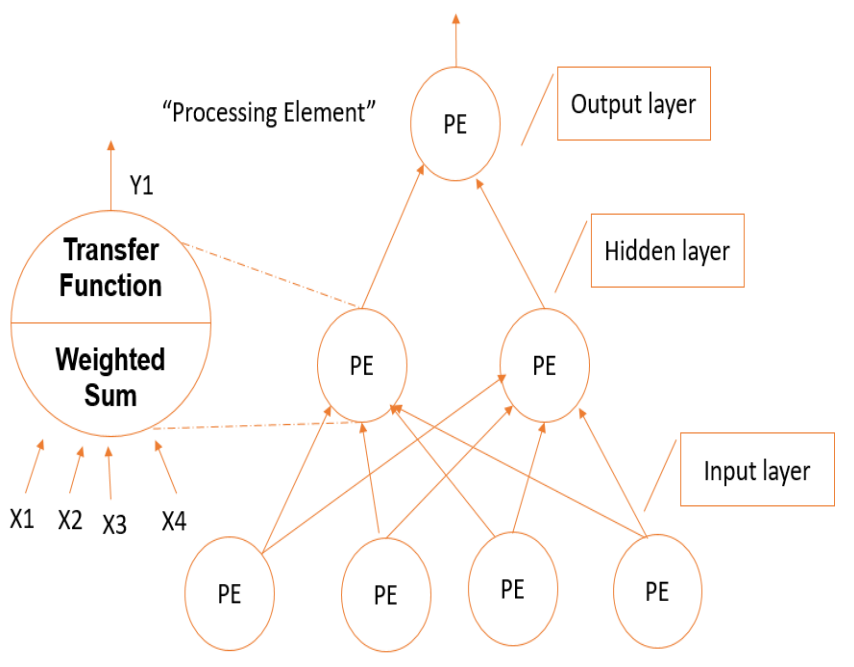

Fig. 5. Neural Network with Layers

\section{CHURN PREDICTION USING PREDICTIVE ANALYSIS MODELING}

The conceptual infrastructure from conception to ultimate testing is drawing in figure: i. The data is collected from interest group according to the behavior attributes that clearly distinguish churners from non-churners

ii. The variables are extracted, transform from given data set items

iii. The data are then analyzed for the functionality for predefine technique for our specific application. Most famous are association, clustering or prediction

iv. The model is build according to statistical or machine learning technique that is set theory, Bayesian classification, neural network, logistic regression, decision tree, genetic algorithm, linear regression etc.,

v. The already developed model is passed through testing and evaluation phase to check the accuracy.

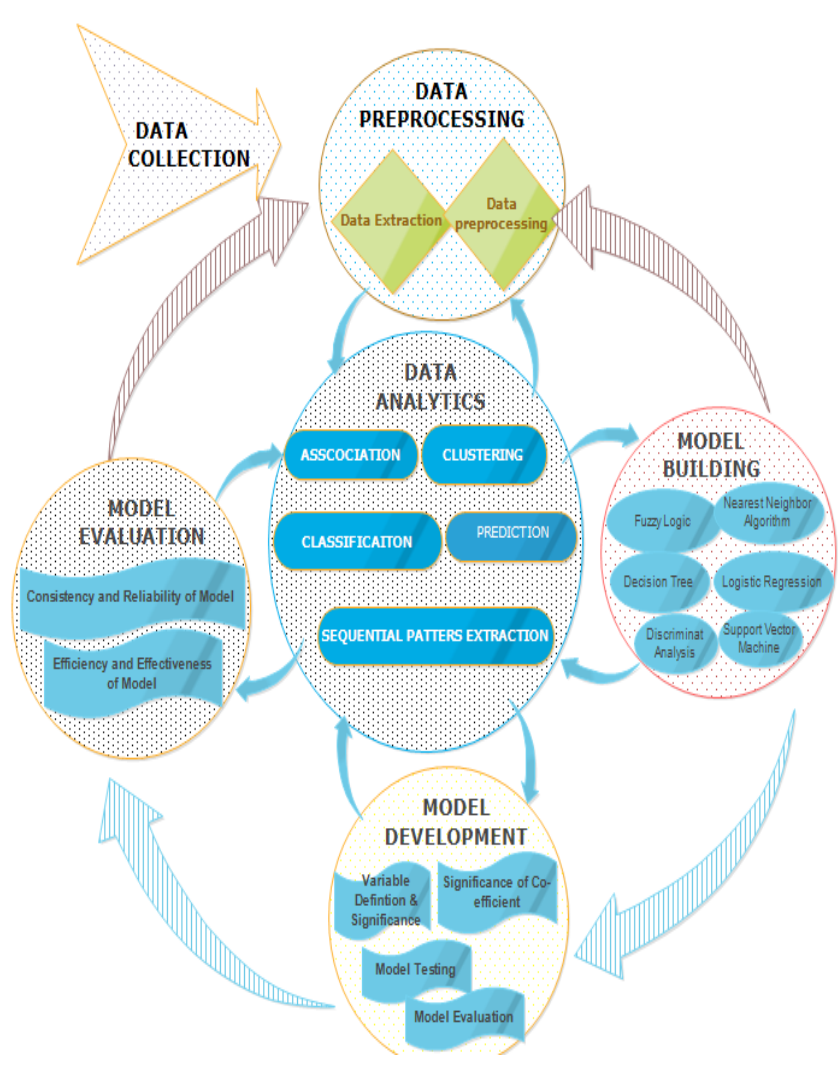

Fig. 6. The conceptual Frame Work

\section{PERFORMANCE METRICS}

Evaluation of a particular model is performed through performance metric which determine how effective is our classifier for a certain churn prediction. The metrics are explained as below:

\section{A. Confusion Metrics}


Model accuracy and correctness can be found through confusion metrics used frequently for classification problem. The matrix is comprised of two dimensional table (predicted and actual) along with set of classes. The actual class is represented in columns while predicted class is rows.

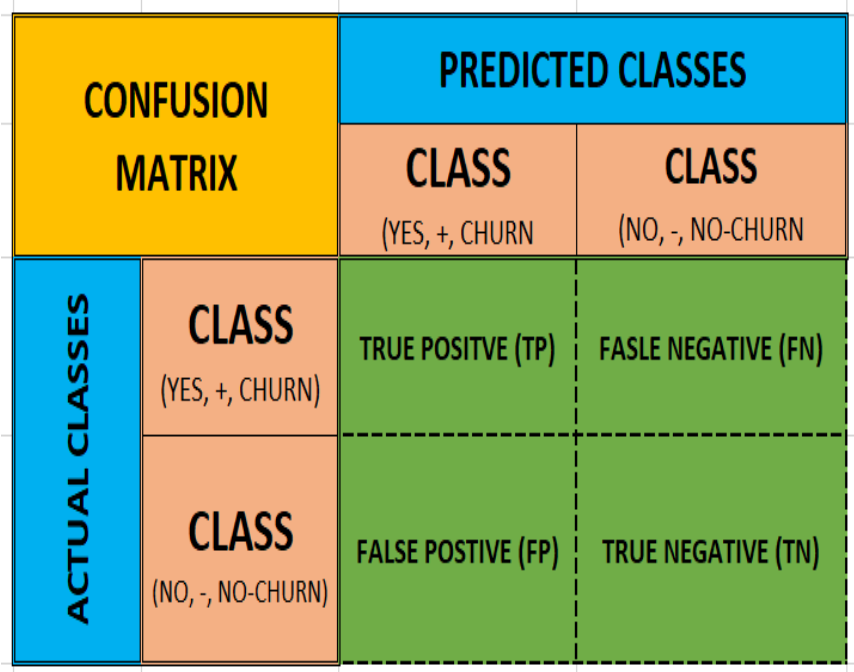

TABLE 2: Confusion Matrix

The confusion matrix will result true positive (TP) when both predicted and actual class give true values, true negative (TN) when both predicted and actual class give false values, false positive (FP) when actual class is false and predicted is true and false negative (FN) is status of CM when actual class is true and predicted is false.

\section{B. Accuracy}

This is mostly used in classification problem for correct prediction of over all kind of prediction. Mathematically, it is represented as below:

\section{Precision}

$$
\text { Accuracy }=\frac{\mathrm{TP}+\mathrm{TN}}{\mathrm{TP}+\mathrm{FP}+\mathrm{FN}+\mathrm{TN}}
$$

The measurement to explain the proportion of values having problem in reality. Mathematically, it is represented as below:

\section{Recall}

$$
\text { Precision }=\frac{\mathrm{TP}}{\mathrm{TP}+\mathrm{FP}}
$$

The measurement of proportion of real cases actually predicted by model. Mathematically, it is represented as below:

\section{E. Specificity}

$$
\text { Recall }=\frac{\mathrm{TP}}{\mathrm{TP}+\mathrm{FN}}
$$

The proportion of true results predicted as true by a particular model

$$
\text { Specificity }=\frac{\mathrm{TN}}{\mathrm{TN}+\mathrm{FP}}
$$

(The exact opposite of recall is specificity)

\section{F. F1-Score}

The score a model obtained on the combination of both precession and recall. Mathematically, it is represented as below:

$$
\text { F1 Score }=\frac{2 * \text { Precision } * \text { Recall }}{\text { Precision }+ \text { Recall }}
$$

\section{G. Receiver operating Characteristic Curve(ROC)}

The curve represent true positive rate and false positive rate on $\mathrm{x}$-axis and $\mathrm{y}$-axis to determine both benefits and costs respectively on 1 learner scale. After having compromise between benefits and cost, this curve represent the connection churners ration identified correctly as churner while nonchurner ration mistakenly recorded as churner.

Best Performance out of model is achieved when the cure passes thourgh or near to 0 or 1 and sensitivity and specificity would reach to $100 \%$.In some instances even in logistic regression, instead binary value other discreate values are derived.

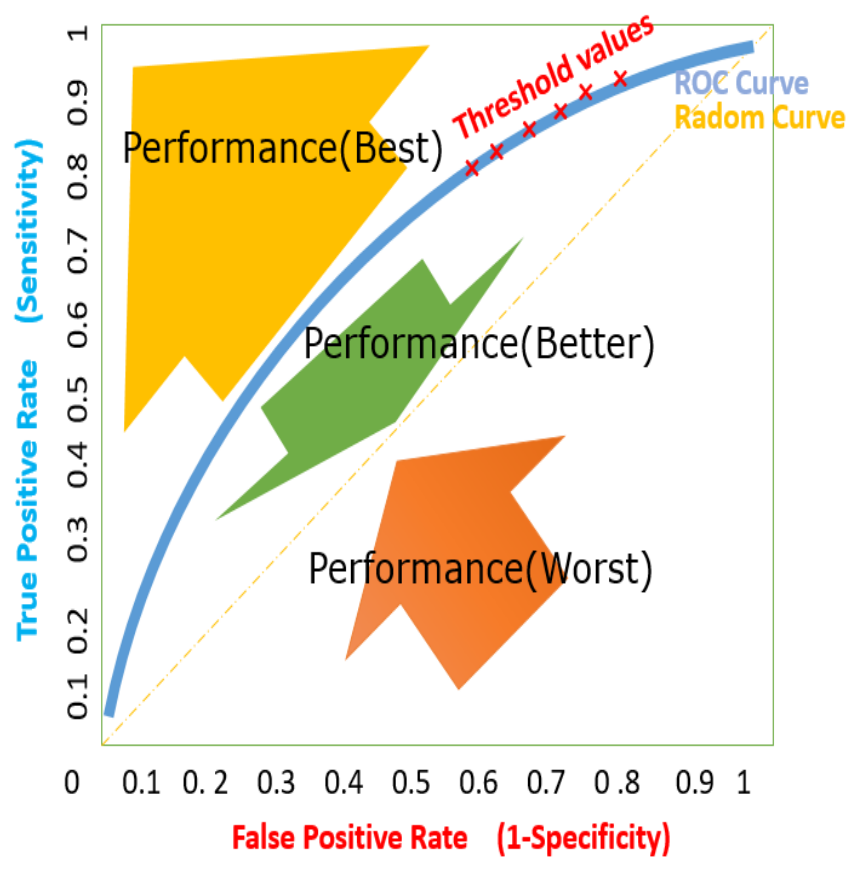

Fig. 7. ReceiverOperatingCharacteristic(ROC) Curve 
The total area of the is divided by Random line, so space above the line are considered as beter performance area and on the line are random guessing classifiers. The aea below the ROC curve ae called area under the curve (AUC) ranges from 0.0 to 1.0 so AUC value greater than 0.5 will give out better performance area.

\section{H. Lift Curve}

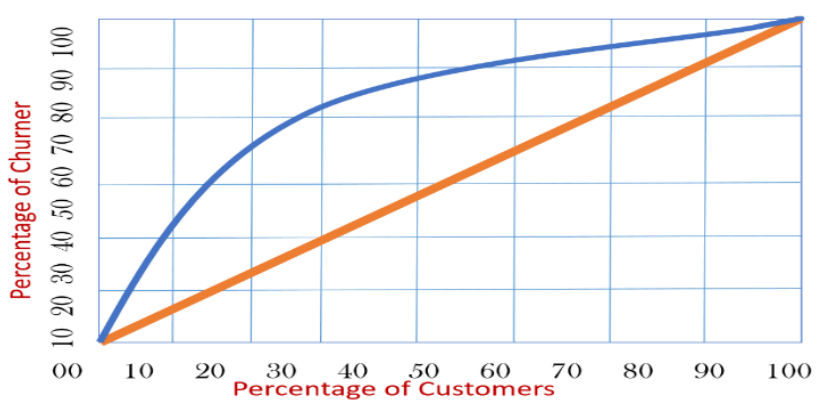

Fig. 8. The Lift Curve

This curve is used in the situation when true positive (TP) can not be determined due to non-availability of classification instances where ROC curve is unable to perform its due role to determine the accuracy of models. The prediction performance measuring scale used in lift curve is deciles. The first two deciles predict the information of $50 \%$ churners and first five deciles provide data of about $90 \%$ churner. The main reason behind the popularity of this metric is relative probability of churner and target for churners become more narrow when top two deciles are present for retention.

\section{PROPOSITION OF MODEL FOR CHURN PREDICTION}

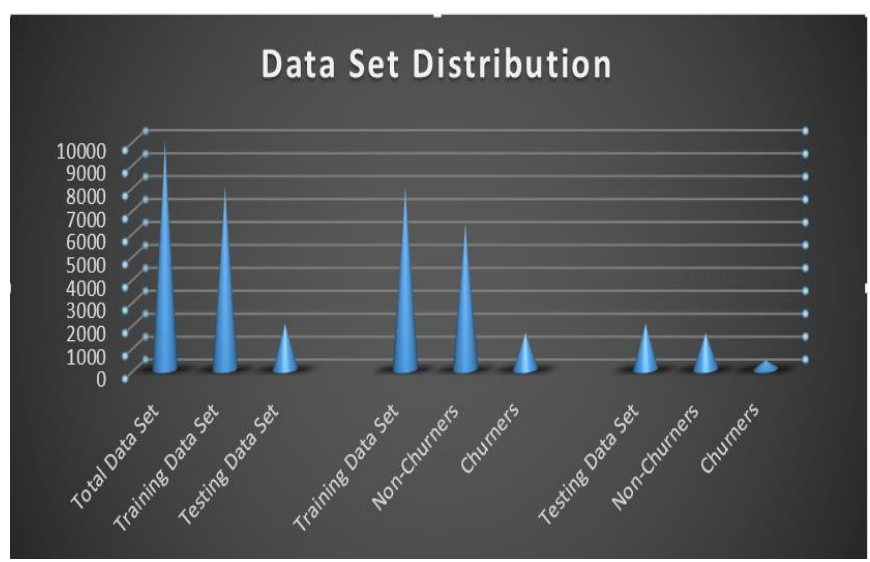

Fig. 9. The Distribution of Data Set

TABLE 3: Details of Data set Attributes

\begin{tabular}{|c|c|c|}
\hline Attributes & Description of Attributes & Data Type \\
\hline age & age into five groups & Nominal \\
\hline gen & gender & Ordinal \\
\hline mar_st & marital status & Ordinal \\
\hline av_in_sm & avg. incoming call from same company & Scale \\
\hline av_ou_sm & avg. outgoing call from same company & Scale \\
\hline av_al_sm & avg.all call from same company & Scale \\
\hline av_in_an & avg.incoming call from another company & Scale \\
\hline av_ou_an & avg.outgoing call from another company & Scale \\
\hline av_al_an & avg.all call from another company & Scale \\
\hline av_in_s_a & avg. incoming call either of same or another company & Scale \\
\hline av_ou_s_a & avg.outgoing call from either same or another company & Scale \\
\hline av_al_s_a & avg. all call either of same or another company & Scale \\
\hline av_i_o_s_a & avg.of incoming\&outgoing call either from same or another company & Scale \\
\hline ch_mnth & change in use from one month to another month during observation period & Scale \\
\hline av_sms & avg. number of short messaging service during observation period & Scale \\
\hline av_rev & avg. monthly revenue & Scale \\
\hline vo_\&_da & both voice and data service & Scale \\
\hline vo_\&_iptv & both voice and multimedia service & Scale \\
\hline vo_da_iptv & Triple play service & Scale \\
\hline av_cuscar & avg.calls generated from customer service representative & Scale \\
\hline av_comp & avg. complaints registered & Scale \\
\hline ch_pack & changes in packages & Scale \\
\hline per_bw_cal & time period between two call & Scale \\
\hline churn & churn (yes/no) & Nominal \\
\hline
\end{tabular}


For analysis of data dataming tool (open source) tool called WEKA was used.Pakistan Telecommunication company limited is national internet service provider and backone for telecom infrastructure despite a number of telcom corporations in entire country market. These services are GSM, LTE, CDMA,HSPA+, IPTV. Total $26 \%$ share of the company has been sold to Etisalate Telecommunicaitons while remain $74 \%$ is still owned by state.

Data of almost 10,000 instances have been collected from PTCL which is divided as training and testing data as 8000 and 2000 respecitvely. Similary 6400(80\%) instances are labeled as non-churner and 1600 cases labeled as churner.

Out of total 2000 instances from testing data set, 1600 cases are considered as non-churner while the remaining 400 cases are taken as churners. The distribution of entire data set is illustrated in Figure-9 for pictoral view.

The given data is divided into 24 attributes according to available filed taken from company for analysis illustrated in Table-4. The first section is covered by demographic information about the customers then voice service in minutes through telephonic call from same network or another network or both.This data is then feed into WEKA software and results are drawn and mentioned in details in Table-5.

\section{A. Results Interpretation}

The result interpretation of classification method and role of churn and non-churner in Decision Tree, Neural Network and Support vector machine is given in Table-5:

\section{B. Accuracy and Error rate Comparison}

By comparing the accuracy in different methodologies used for prediction of customer churn, it is declared that the best result can be extracted from neural network getting $85 \%$ accuracy and $15 \%$ error rate, Support vector machine getting $84 \%$ accuracy and $16 \%$ error rate on second position and decision tree at third position getting $78 \%$ accuracy and $22 \%$ error rate are drawn in line chart as below:

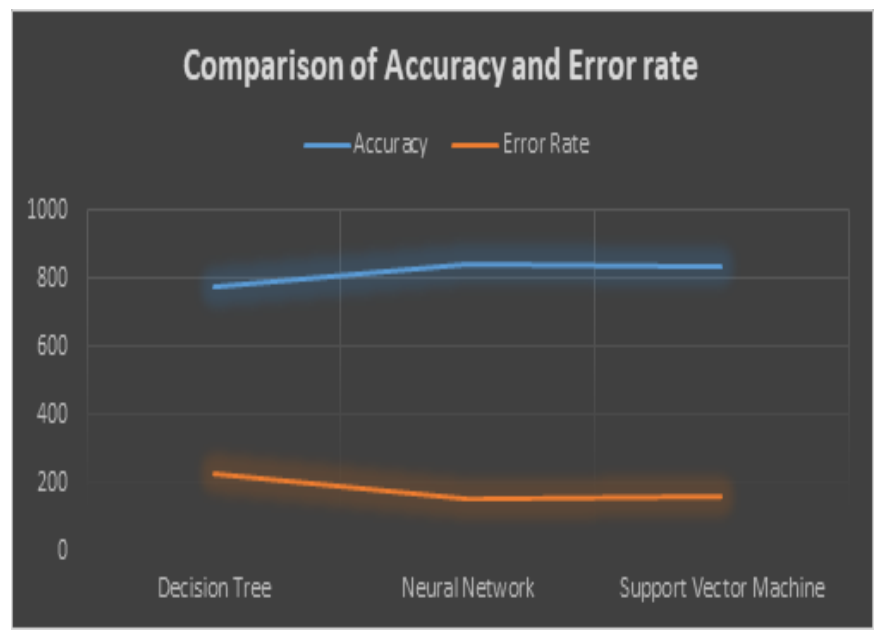

Fig. 10. The Comparision Of Accuracy And Error Rate
TABLE 4: Interpretation of Results

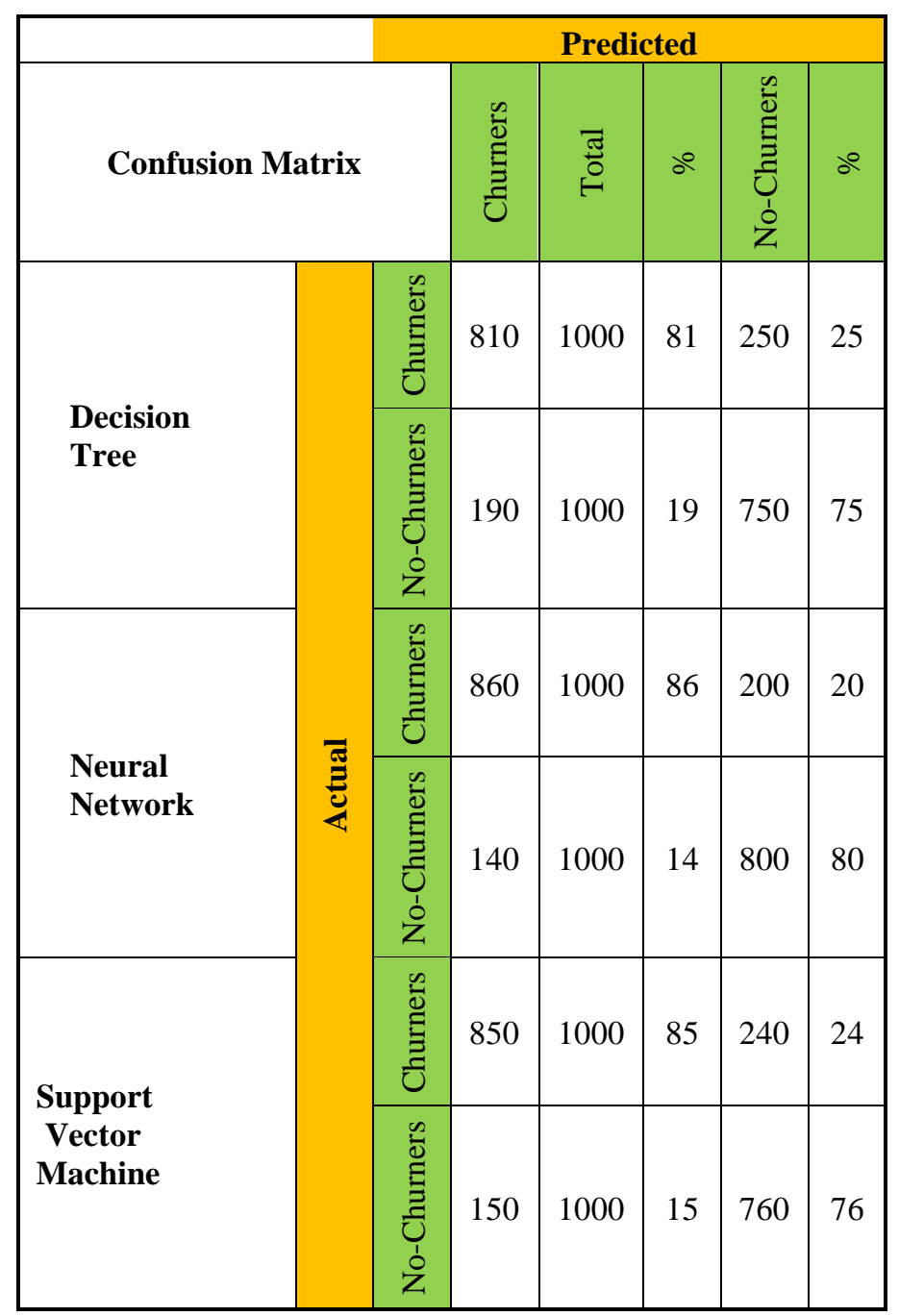

\section{CONCLUSION}

In this paper, all related literature yet available regarding predictive analytics on customer churn is discussed in detail and explained the advantages and disadvantages of each method is mentioned. The sole purpose of this paper is not design any new algorithm for churn prediction but to accumulate and categorized the existing technique already prevailed in the competitive telecom market. The methods along with pictorial representation is furnished with required details, the entire process of data mining from data collection to model evaluation is explained in form of conceptual framework. The metrics to determine the performance of each model is mentioned in details, from confusion metrics, accuracy, precession, recall, specificity, receiver operating characteristic curve and lift curve. A case study on 10, 000 randomly selected customers of Pakistan Telecom Company Limited, one of renown national corporation in Pakistan telecom market, is presented for 
explanation of predictive analytics process. After applying data mining most famous techniques on data set provided and conclude that neural network is method having more accuracy and lower error rate as compare to support vector machine and decision tree. Moreover, the process of churn prediction is explained and cleared from literature review that churn prediction is pre-requisite to initiate any retention campaign and imperative for all sort of service.

\section{REFERENCES}

[1] J. G. Palma and J. E. Stiglitz, "Do nations just get the inequality they deserve? The "Palma Ratio" re-examined," in Inequality and Growth: Patterns and Policy: Springer, 2016, pp. 35-97.

[2] H. G. Mishra, P. K. Sinha, and S. J. J. o. I. B. R. Koul, "Customer dependence and customer loyalty in traditional and modern format stores," vol. 9, no. 1, pp. 59-78, 2017.

[3] R. Gubela, A. Bequé, S. Lessmann, F. J. I. J. o. I. T. Gebert, and D. Making, "Conversion uplift in e-commerce: A systematic benchmark of modeling strategies," vol. 18, no. 03, pp. 747-791, 2019.

[4] P. A. Sarvari, A. Ustundag, and H. J. K. Takci, "Performance evaluation of different customer segmentation approaches based on RFM and demographics analysis," vol. 45, no. 7, pp. 1129-1157, 2016.

[5] K. Coussement, S. Lessmann, and G. J. D. S. S. Verstraeten, "A comparative analysis of data preparation algorithms for customer churn prediction: A case study in the telecommunication industry," vol. 95, pp. 27-36, 2017.

[6] A. Amin, F. Al-Obeidat, B. Shah, A. Adnan, J. Loo, and S. J. J. o. B. R. Anwar, "Customer churn prediction in telecommunication industry using data certainty," vol. 94, pp. 290-301, 2019.

[7] P. Bertens, A. Guitart, P. P. Chen, and Á. Periáñez, "A Machine-Learning Item Recommendation System for Video Games," in 2018 IEEE Conference on Computational Intelligence and Games (CIG), 2018, pp. 1-4: IEEE.

[8] E. Stripling, S. vanden Broucke, K. Antonio, B. Baesens, M. J. S. Snoeck, and E. Computation, "Profit maximizing logistic model for customer churn prediction using genetic algorithms," vol. 40, pp. 116-130, 2018.

[9] M. Azeem, M. Usman, and A. C. M. J. T. S. Fong, "A churn prediction model for prepaid customers in telecom using fuzzy classifiers," vol. 66, no. 4, pp. 603-614, 2017.

[10] M. Óskarsdóttir, C. Bravo, W. Verbeke, C. Sarraute, B. Baesens, and J. J. E. S. w. A. Vanthienen, "Social network analytics for churn prediction in telco: Model building, evaluation and network architecture," vol. 85, pp. 204-220, 2017.

[11] M. A. Kilian, M. Kattenbeck, M. Ferstl, B. Ludwig, and F. J. A. J. o. I. M. Alt, "Towards task-sensitive assistance in public spaces," vol. 71, no. 3, pp. 344-367, 2019.

[12] M.-J. Kim, J. Kim, S.-Y. J. A. P. J. o. I. Park, and Entrepreneurship, "Understanding IPTV churning behaviors: focus on users in South Korea," vol. 11, no. 2, pp. 190-213, 2017.

[13] L. E. Kleivene, "P2P Mobile Payments: Investigating the Factors of Adoption Among Students in Germany," in Omnichannel Branding: Springer, 2018, pp. 45-67.

[14] M. Rafiy, P. J. A. o.-1. P. i. E. Adam, and Informatics, "The Demand of Services for Information Technology Industry in Indonesia," vol. 8, no. 665-2016-45135, pp. 125-132, 2016.

[15] P. Thaichon, K. Sharma, K. Raina, and S. J. A. J. o. B. R. I. Kapoor, "Analysis of consumers' intention values in the choice of a mobile service provider," vol. 6, no. 1, p. 2016, 2016.

[16] Y. Sun, D. Liu, S. Chen, X. Wu, X.-L. Shen, and X. J. C. i. H. B. Zhang, "Understanding users' switching behavior of mobile instant messaging applications: An empirical study from the perspective of push-pullmooring framework," vol. 75, pp. 727-738, 2017.

[17] P. K. Dalvi, S. K. Khandge, A. Deomore, A. Bankar, and V. Kanade, "Analysis of customer churn prediction in telecom industry using decision trees and logistic regression," in 2016 Symposium on Colossal Data Analysis and Networking (CDAN), 2016, pp. 1-4: IEEE.
[18] O. C. Ojiaku and A. J. G. B. R. Osarenkhoe, "Determinants of customers' brand choice and continuance intentions with mobile data service provider: The role of past experience," vol. 19, no. 6, pp. 1478-1493, 2018.

[19] P. J. a. p. a. Sundsøy, "Big Data for Social Sciences: Measuring patterns of human behavior through large-scale mobile phone data," 2017.

[20] A. De Caigny, K. Coussement, and K. W. J. E. J. o. O. R. De Bock, "A new hybrid classification algorithm for customer churn prediction based on logistic regression and decision trees," vol. 269 , no. 2, pp. 760-772, 2018.

[21] A. O. Ibitoye, O. F. J. I. J. o. I. Onifade, Business, and Management, "Customer churn predictive analytics using relative churn fuzzy featureweight model in telecoms," vol. 11, no. 3, pp. 163-175, 2019.

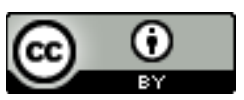

Journal of Applied and Emerging
Sciences by BUITEMS is licensed under a Creative
Commons Attribution 4.0 International License.

